\title{
Limiting ozone vegetation damage
}

Surface ozone arises through complex photochemical reactions of carbon monoxide, methane, other volatile organic compounds and nitrous oxides in sunlight. In addition to the widely known effects on air quality and human health, rising concentrations of ozone also have deleterious impacts on vegetation growth, influencing carbon sequestration. In Europe, eastern China and the eastern United States, for example, high levels of surface ozone pollution have been connected to decreased gross primary production (GPP). Surface ozone therefore threatens natural processes which act to limit

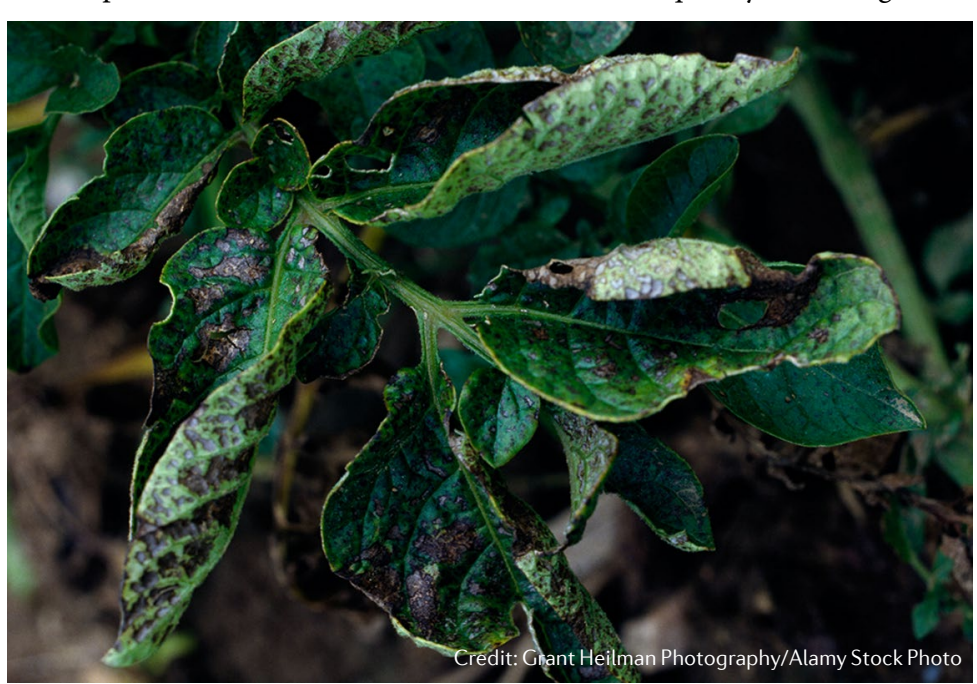

anthropogenic warming, motivating the need for ozone reductions. However, it is unknown which source sectors should be targeted for such mitigation efforts.

Nadine Unger from the University of Exeter, UK, and colleagues, ran a global Earth System Model (NASA ModelE2-YIBs) with seven mitigation scenarios wherein a $50 \%$ reduction in ozone precursor emissions is applied to the agriculture, domestic, energy, industry, road transportation, waste and shipping sectors. At the global scale, it is found that the transport and energy sectors offer the largest reductions in surface ozone, consequently increasing GPP

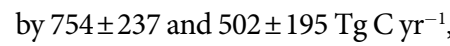
respectively. Regionally, the transport and energy sectors mitigate $~ 16 \%$ of ozone-related GPP losses in Europe, the eastern US and eastern Asia, with the latter also profiting from reductions in industrial and agricultural sector emissions. Benefits are particularly pronounced for C3 cropland and C3 and C4 grassland ecosystems, linked to differential tolerance thresholds for detoxification from ozone damage.

The transport and energy sectors therefore offer an ideal target for initial efforts to constrain ozonerelated GPP losses. However, 50\% reductions in ozone precursors will be ambitious. Nevertheless, given that the transport and energy sectors simultaneously account for a large proportion of global $\mathrm{CO}_{2}$ emissions, any mitigation measures to constrain anthropogenic warming will have the co-benefit of reducing surface ozone and, in turn, enhance plant (and human) health, contributing to negative $\mathrm{CO}_{2}$ emissions. These coupled interactions and feedbacks clearly showcase the value of mitigating surface ozone.

Graham Simpkins

ORIGINAL ARTICLE Unger, N. et al. Mitigation of ozone damage to the world's land ecosystem by source sector. Nat. Clim. Change https://doi.org/ 10.1038/s41558-019-0678-3 (2020) 